\title{
Conjugate natural convection in a square cavity with finite thickness horizontal walls
}

\author{
Moghtada Mobedi \\ Mechanical Engineering Department, Izmir Institute of Technology, Urla 35430, Izmir, Turkey
}

Available online 20 November 2007

\begin{abstract}
The effect of conduction of horizontal walls on natural convection heat transfer in a square cavity is numerically investigated. The vertical walls of the cavity are at different constant temperatures while the outer surfaces of horizontal walls are insulated. A code based on vorticity-stream function is written to solve the governing equations simultaneously over the entire computational domain. The dimensionless wall thickness of cavity is taken as 0.1 . The steady state results are obtained for wide ranges of Rayleigh number $\left(10^{3}<R a<10^{6}\right)$ and thermal conductivity ratio $(0<K<50)$. The variation of heat transfer rate through the cavity and horizontal walls with Rayleigh number and conductivity ratio is analyzed. It is found that although the horizontal walls do not directly reduce temperature difference between the vertical walls of cavity, they decrease heat transfer rate across the cavity particularly for high values of Rayleigh number and thermal conductivity ratio. Heatline visualization technique is a useful application for conjugate heat transfer problems as shown in this study.
\end{abstract}

(C) 2007 Elsevier Ltd. All rights reserved.

Keywords: Conjugate natural convection; Cavity, Conductivity ratio

\section{Introduction}

Natural convection heat transfer in a cavity is investigated by many researchers due to its wide application areas. Studies on heat and fluid flow in the cavities are continued since in addition to Rayleigh and Prandtl numbers, heat transfer through a cavity is influenced by parameters such as wall boundary conditions, inclination, aspect ratio, cavity geometry and bodies located in a cavity. Kalabin et al. [1,2] investigated the problems of natural convection heat transfer in a cavity with timevarying side-wall temperature and heat transfer from the cold wall of a square cavity to the hot one by oscillatory natural convection. Natural convection in a square cavity with spatial side-wall temperature variation was investigated by Saeid and Yaacob [3]. They investigated the effects of amplitude and the wave number of the heated side-wall temperature variation on the natural convection in the cavity. The effect of length and inclination of a thin fin on natural convection in a square cavity was studied by Ben-Nakhi and Chamkha [4]. For irregular shaped enclosure, Varol et al. [5] employed central difference scheme for numerical solution of laminar natural convection. Lo et al. [6] improved solution method by generalized differential quadrature method to obtain accurate results by much coarse mesh.

\footnotetext{
is Communicated by W.J. Minkowycz.

E-mail address: moghtadamobedi@iyte.edu.tr.
} 


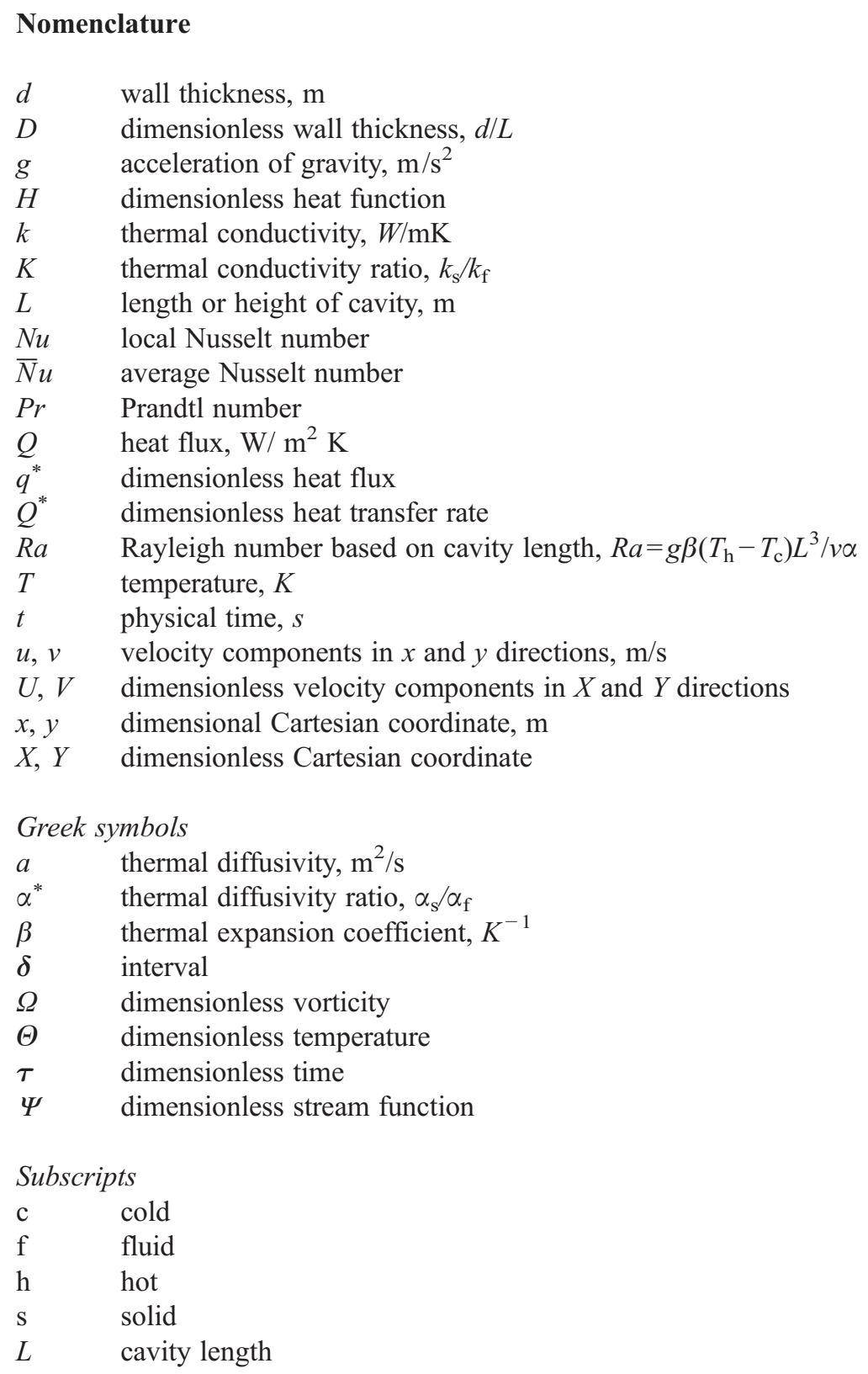

The influence of wall heat conduction on natural convection heat transfer in a cavity has gained attention of researchers in recent years. Kim and Viskanta [7,8] performed experimental and numerical studies on natural convection in a square cavity having four walls with finite thickness. The effects of wall heat conduction and surface radiation on natural convection in a two dimensional rectangular cavity were also studied by Kim and Viskanta [9]. Wu et al. [10] experimentally studied the effects of top and bottom walls temperature on the natural convection heat transfer in an airfilled square cavity driven by a difference in the vertical wall temperatures. Kaminski and Prakash [11] performed a numerical study on conjugate natural convection heat transfer in a square cavity with a finite thickness vertical wall to compare different models of wall heat conduction. A finite element study on natural convection in a square cavity with a conducting vertical wall was done by Misra and Sarkar [12]. Liaqat and Baytas [13] numerically studied natural convection flow in a square enclosure containing a volumetric source which is distributed within the cavity. The influence of wall conduction on natural convection in an inclined square cavity was researched by Acharya and Tsang 


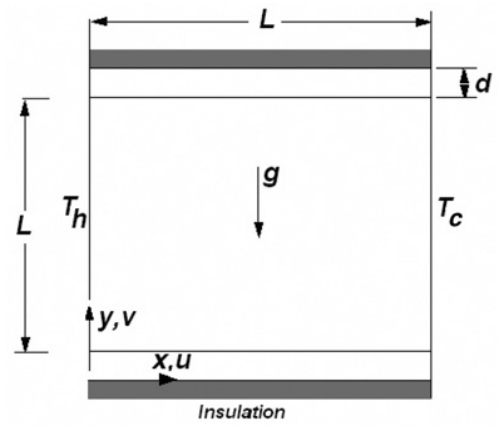

Fig. 1. Schematic view of the considered square cavity.

[14]. Du and Bilgen [15] performed a study on an enclosure which consists of a finite thickness conducting wall with a uniform heat input, an insulated vertical wall and two horizontal walls at a heat sink temperature. The effects of Ra number, dimensionless conductivity ratio, dimensionless wall width and inclination angle on the laminar natural convection in an inclined enclosure bounded by a solid wall were investigated by Yedder and Bilgen [16]. Saeid [17] performed a numerical study to investigate the effect of heat conduction of a vertical wall on convection in a porous enclosure. Natural convection heat transfer in a square cavity filled with porous medium and has two finite thickness horizontal walls was numerically investigated by Baytas et al. [18]. Chang and Lin [19] studied wall heat conduction effect on natural convection in an enclosure filled with a non-Darcian porous medium.

Literature survey shows that the wall heat conduction can play an important role on natural convection in a cavity. The measure of conduction effect depends on the dimensionless governing parameters which are $R a$ number, thermal conductivity and diffusivity ratios, dimensionless wall thickness and $\operatorname{Pr}$ number. Most of studies in literature were performed on cavity with one vertical thick wall or four thick walls which surround the cavity. The influence of vertical wall heat conduction on natural convection in a cavity may be predicted since it reduces temperature difference across the cavity. However, the effect of horizontal walls is not clearly known. The main intent of this study is to investigate the influence of horizontal walls conductance on natural convection heat transfer in an air-filled square cavity. The isotherms, streamlines and heatlines are drawn to exhibit the mechanism of heat and fluid flow in the cavity.

\section{The considered problem}

The cavity is a square with a side length of $L$ as shown schematically in Fig. 1. The horizontal walls have finite thickness, $d$, and the outer surfaces of horizontal walls are insulated. The vertical walls of the cavity are maintained at different $T_{\mathrm{h}}$ and $T_{\mathrm{c}}$ constant temperatures such that $T_{\mathrm{h}}>T_{\mathrm{c}}$. The heat transfer in the cavity occurs by laminar natural convection. The problem is solved for air with $\operatorname{Pr}=0.71$.

\section{Governing equations and boundary conditions}

The governing equations for the problem are continuity, momentum and energy equations for fluid inside the cavity and heat conduction equation for the horizontal walls. The radiation effect is neglected and Boussinesq approximation was applied. By employing the dimensionless vorticity and stream function parameters, the dimensionless form of governing equations can be written as:

$$
\begin{aligned}
& \frac{\partial \Omega}{\partial \tau}+\frac{\partial U \Omega}{\partial X}+\frac{\partial V \Omega}{\partial Y}=\operatorname{Pr}\left(\frac{\partial^{2} \Omega}{\partial X^{2}}+\frac{\partial^{2} \Omega}{\partial Y^{2}}\right)+R a \cdot \operatorname{Pr} \cdot \frac{\partial \theta_{\mathrm{f}}}{\partial X} \\
& \frac{\partial^{2} \Psi}{\partial X^{2}}+\frac{\partial^{2} \Psi}{\partial Y^{2}}=-\Omega \\
& \frac{\partial \theta_{\mathrm{f}}}{\partial \tau}+\frac{\partial U \theta_{\mathrm{f}}}{\partial X}+\frac{\partial V \theta_{\mathrm{f}}}{\partial Y}+\frac{\partial^{2} \theta_{\mathrm{f}}}{\partial X^{2}}+\frac{\partial^{2} \theta_{\mathrm{f}}}{\partial Y^{2}}
\end{aligned}
$$


where $U, V, \theta_{\mathrm{f}}, \tau, X$ and $Y$ are dimensionless parameters:

$$
X=x / L, Y=y / L, U=u L / \alpha_{\mathrm{f}}, V=v L / \alpha_{\mathrm{f}}, \theta_{\mathrm{f}}=\left(T_{\mathrm{f}}-T_{\mathrm{c}}\right) /\left(T_{\mathrm{h}}-T_{\mathrm{c}}\right), \tau=\alpha_{\mathrm{f}} t / L^{2} .
$$

The dimensionless vorticity and stream function are defined as $\Omega=\partial V / \partial X-\partial U / \partial Y, U=\partial \Psi / \partial Y, V=-\partial \Psi / \partial X$. Based on the defined dimensionless parameters, the dimensionless form of heat conduction equation for the horizontal walls becomes as:

$$
\frac{\partial \theta_{\mathrm{S}}}{\partial \tau}=\alpha^{*}\left(\frac{\partial^{2} \theta_{\mathrm{S}}}{\partial X^{2}}+\frac{\partial^{2} \theta_{\mathrm{S}}}{\partial Y^{2}}\right)
$$

where $q_{\mathrm{s}}=\left(T_{\mathrm{S}}-T_{\mathrm{c}}\right) /\left(T_{\mathrm{h}}-T_{\mathrm{c}}\right)$ and $a^{*}$ are horizontal wall dimensionless temperature and thermal diffusivity ratio respectively. The boundary conditions for the domain which is shown in Fig. 1 can be written as follows:

$$
\begin{array}{ll}
Y=0, Y=1+2 D & \Omega=\Psi=\frac{\partial \theta_{\mathrm{S}}}{\partial Y}=0 \\
X=0, X=1 & \Omega=\frac{\partial V}{\partial X}, \Psi=0, \theta(0, Y, \tau)=1, \theta(1, Y, \tau)=0 \\
Y=D, Y=1+D & \Omega=-\frac{\partial U}{\partial Y}, \Psi=0, \theta_{\mathrm{S}}=\theta_{\mathrm{f}} \text { and }\left.K \frac{\partial \theta_{\mathrm{S}}}{\partial Y}\right|_{\mathrm{s}}=\left.\frac{\partial \theta_{\mathrm{f}}}{\partial Y}\right|_{\mathrm{f}} .
\end{array}
$$

The initial values for dimensionless temperature, vorticity and stream function are assigned as zero. The dimensionless forms of the governing equations and boundary conditions reduce number of independent dimensionless parameters to five which are $R a, \operatorname{Pr}, K, \alpha^{*}$ and $D$. The thermal diffusivity ratio does not play role on the steady state results. The present study is performed for air with $P r=0.71$ and the horizontal wall thickness is not changed. Thus, the effects of $R a$ number and thermal conductivity ratio are investigated in the present study.

The following equation is solved to obtain dimensionless heat function [20]:

$$
\frac{\partial^{2} H}{\partial X^{2}}+\frac{\partial^{2} H}{\partial Y^{2}}=\frac{\partial U \theta}{\partial \mathrm{Y}}-\frac{\partial V \theta}{\partial \mathrm{X}} .
$$

The dimensionless heat function in differential form is defined as:

$$
-\frac{\partial H}{\partial X}=V \theta-\frac{\partial \theta}{\partial Y}, \frac{\partial H}{\partial Y}=U \theta-\frac{\partial \theta}{\partial X} .
$$

The Eq. (9), which is valid for both fluid and solid regions, can be numerically solved. The boundary conditions for Eq. (9) are obtained from the integration of Eq. (10) along the considered boundary. For example, dimensionless heat function values at $X=0$ and $Y=1+D$ boundaries can be determined as:

$$
\begin{array}{ll}
\text { At } X=0 \text { and } 0 \leq Y \leq D & H(0, Y)=H(0,0)-\int_{0}^{D} K \frac{\partial \theta_{\mathrm{s}}}{\partial X} d Y \\
\text { At } X=0 \text { and } D<Y \leq 1+D & H(0, Y)=H(0, D)-\int_{D}^{1+D} \frac{\partial \theta_{\mathrm{f}}}{\partial X} d Y \\
\text { At } X=0 \text { and } D<Y \leq 1+2 D & H(0, Y)=H(0,1+D)-\int_{1+D}^{1+2 D} K \frac{\partial \theta_{\mathrm{s}}}{\partial X} d Y \\
\text { At } Y=1+D \text { and } 0<X \leq 1 & \frac{\partial H}{\partial X}=\frac{\partial \theta_{\mathrm{f}}}{\partial Y}
\end{array}
$$

where at $Y=0$ the value of heat function is zero, $H(X, 0)=0$.

\section{Solution procedure}

The set of governing equations is numerically solved by finite difference method $[21,22]$. The solution procedure is continued until the steady state is reached. The finite difference forms of diffusion and convection terms are written based 
Table 1

Comparison of the present numerical results with Vahl Davis solution [24]

\begin{tabular}{lllcc}
\hline$R a$ & \multicolumn{2}{l}{ Vahl Davis } & & \multicolumn{2}{c}{ Present Study } \\
\cline { 2 - 3 } & $|\Psi|_{\max }$ & $\bar{N} u$ & $\left.1 \Psi\right|_{\max }$ & $\bar{N} u$ \\
\hline $10^{3}$ & - & 1.118 & 1.174 & 1.114 \\
$10^{4}$ & - & 2.243 & 5.109 & 2.240 \\
$10^{5}$ & 9.612 & 4.519 & 9.693 & 4.510 \\
$10^{6}$ & 16.750 & 8.800 & 16.916 & 8.803 \\
\hline
\end{tabular}

on three points central difference which has second order accuracy. The vorticity, stream function and energy equations are solved for the entire computational domain. The value of Prandtl number for flow region is assigned as $P r=0.71$ while it is changed to $P r=10^{20}$ for the solid region. This change of Prandtl number value causes the vorticity, stream function and velocity values in the horizontal walls are calculated as zero and consequently the energy equation of fluid flow is converted to the heat conduction equation for the solid wall. The conservation of energy between solid and fluid is used to calculate solid-fluid interface temperature. The backward and forward differences are used to determine temperature gradients in the solid and fluid sides of interface. The values of vorticity at the solid boundaries are calculated by using the relation developed by Wong and Baker [23]. The convergence criterion for the solution procedure is defined as:

$$
\frac{\sum\left|\theta^{n+1}-\theta^{n}\right|}{\Delta \tau \sum \theta^{n}} \leq 5 \times 10^{-4} \text {. }
$$

The local and average $N u$ number values at $X=0$ surface are calculated by the following equations:

$$
\begin{aligned}
& q_{\mathrm{f}}^{*}=\frac{q_{\mathrm{f}} L}{k_{\mathrm{f}}\left(T_{\mathrm{h}}-T_{\mathrm{c}}\right)}=N u_{\mathrm{f}}=-\left.\frac{\partial \theta_{\mathrm{f}}}{\partial X}\right|_{X=0} \\
& Q_{\mathrm{f}}^{*}=\bar{N} u_{\mathrm{f}}=\int_{Y=D}^{Y=1+D} N u_{\mathrm{f}} \mathrm{d} Y .
\end{aligned}
$$

The dimensionless heat flux and heat transfer rate from horizontal walls at $X=0$ surfaces are defined as:

$$
\begin{aligned}
& q_{\mathrm{s}}^{*}=\frac{q_{\mathrm{s}} L}{k_{\mathrm{s}}\left(T_{\mathrm{h}}-T_{\mathrm{c}}\right)}=-\left.\frac{\partial \theta_{\mathrm{S}}}{\partial X}\right|_{X=0} \\
& Q_{\mathrm{s}}^{*}=\int_{Y=0}^{Y=D} q_{\mathrm{s}}^{*} \mathrm{~d} Y+\int_{Y=1+D}^{Y=1+2 D} q_{\mathrm{s}}^{*} \mathrm{~d} Y .
\end{aligned}
$$

Non-uniform mesh grid sizes were used both for fluid and solid regions. The grid sizes were selected fine near walls. They were expanded continuously towards the center of cavity. The number of nodes in $X$ and $Y$ directions was

Table 2

Comparison between the obtained results with Kaminski et al. solution [11]

\begin{tabular}{llll}
\hline$R a$ & & $\frac{\text { Kaminski et al. }}{\bar{N} u_{\mathrm{f}}}$ & $\frac{\text { Present Study }}{\bar{N} u_{\mathrm{f}}}$ \\
\hline $7.1 \times 10^{2}$ & & 0.87 \\
$7.1 \times 10^{4}$ & $K=1$ & 1.06 & 0.866 \\
& $K=\infty$ & 2.08 & 4.062 \\
$7.1 \times 10^{5}$ & $K=1$ & 2.074 \\
& $K=\infty$ & 7.034 \\
\hline
\end{tabular}



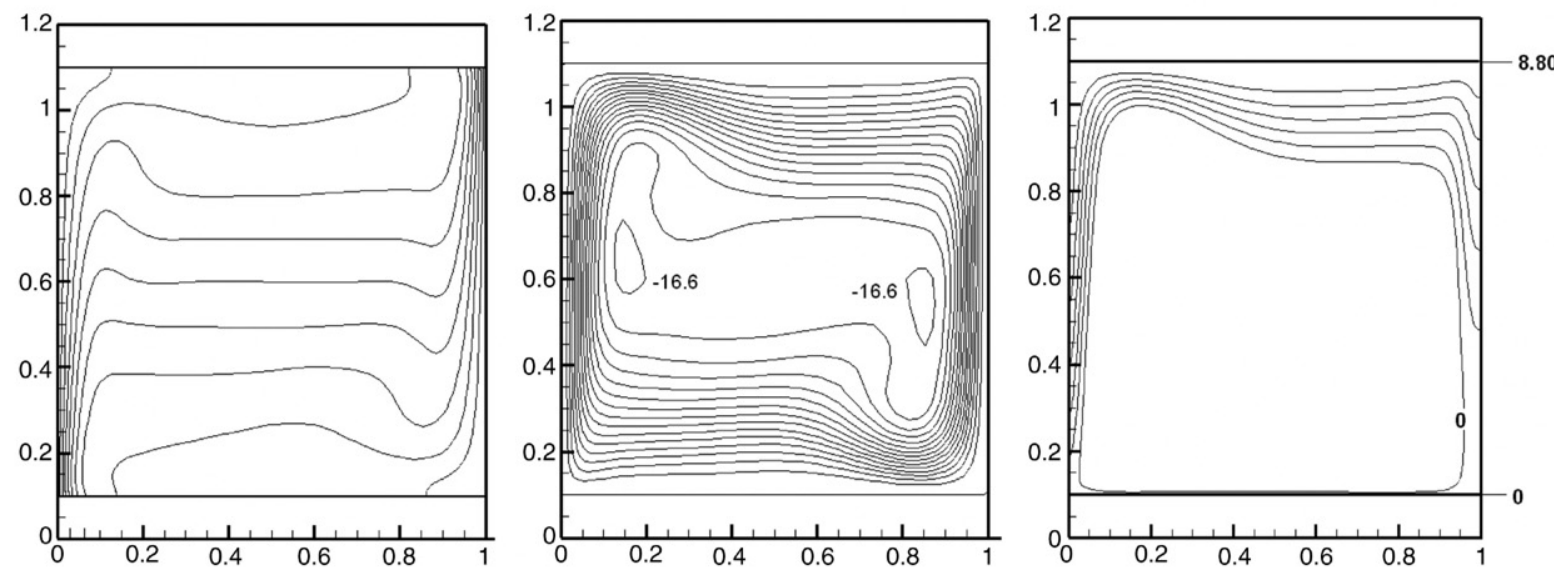

a)
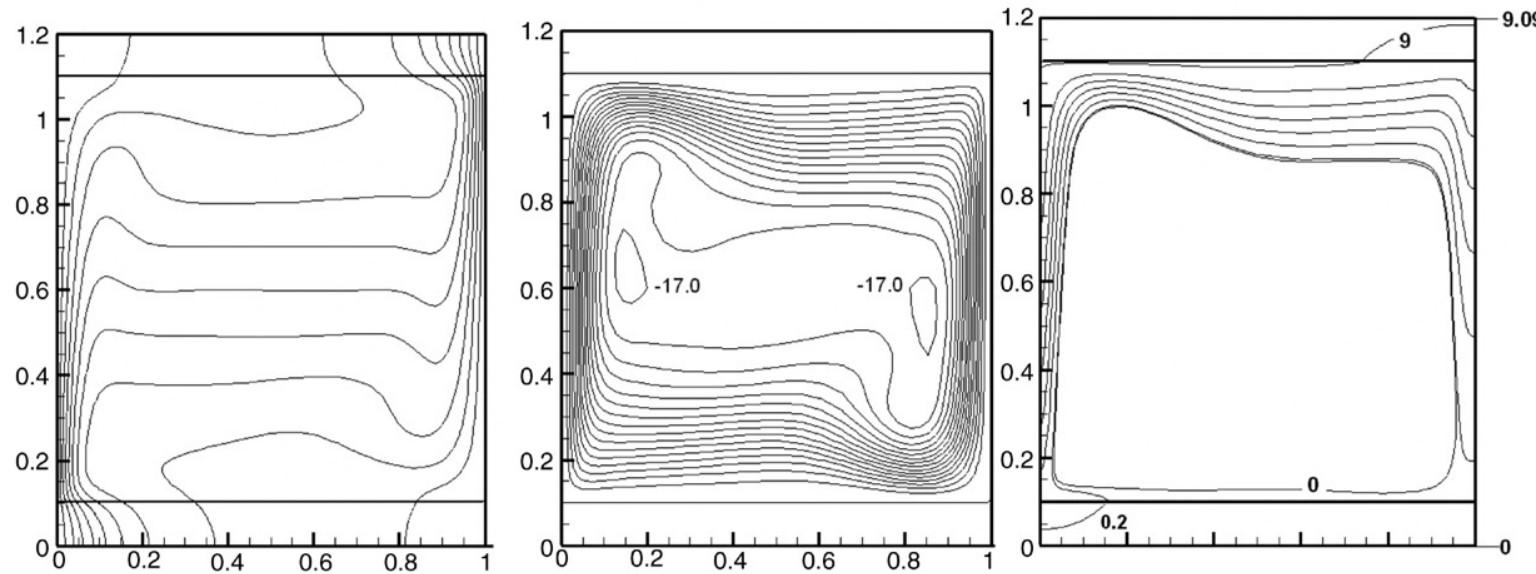

b)
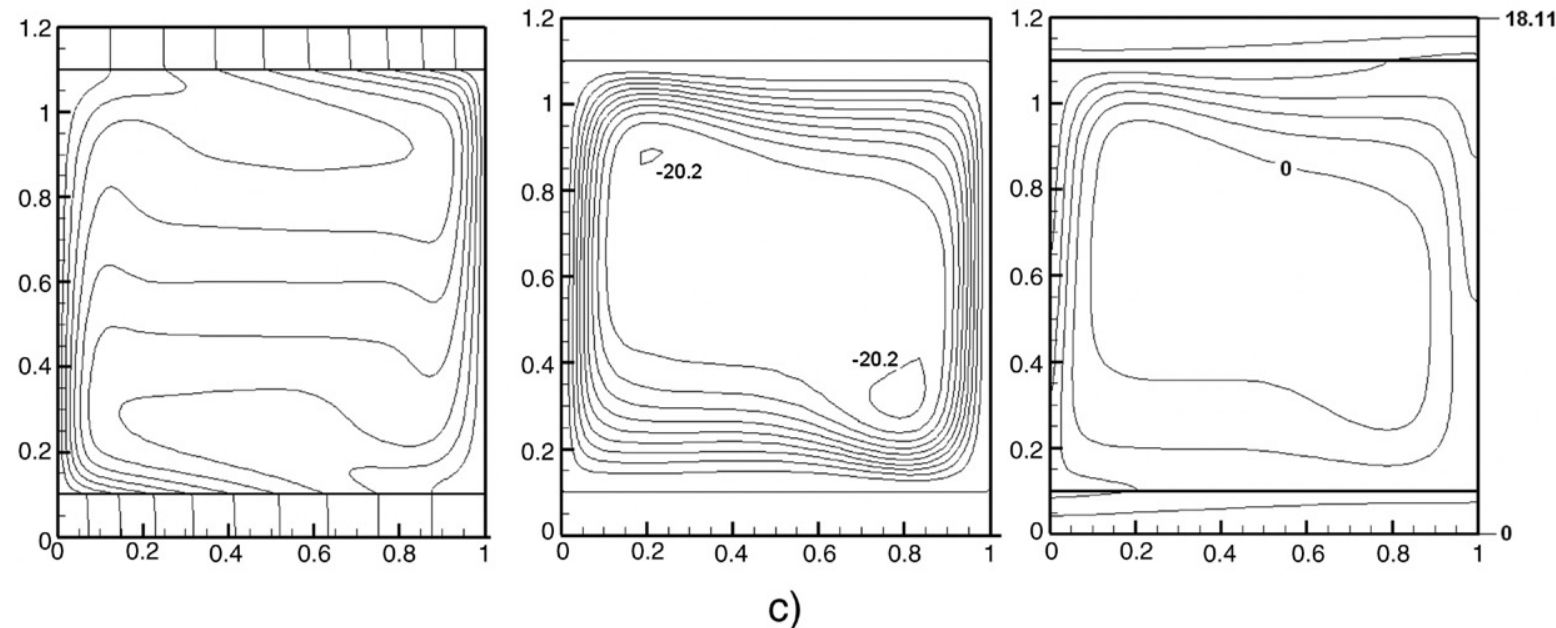

Fig. 2. Isotherms, streamlines and heatlines in the cavity with $R a=10^{6}$, a) $\left.K=0, \delta \theta=0.1, \delta \Psi=1.107, \delta H=1.47 \mathrm{~b}\right) K=1, \delta \theta=0.1, \delta \Psi=1.062$, $\delta H=1.51(H=0.2$ and 9 were added) c) $K=50, \delta \theta=0.1, \delta \Psi=2.02, \delta H=3.02$. 
$80 \times 140$ in which $80 \times 60$ nodes were used in the upper and lower horizontal walls. The smallest grid spacing, which was in the fluid region near to the solid walls, was 0.0005 .

\subsection{Validation of the results}

In order to validate the employed method and check the code, results for pure natural convection in an air-filled square cavity were obtained and compared with the benchmark solution of Vahl Davis [24]. Table 1 shows the comparison between two solutions. The results for problem which was studied by Kaminski and Prakash [16] were also obtained. The comparison between the obtained numerical results and the solution of Kaminski and Prakash is shown in Table 2. A good agreement between the obtained and reported results can be observed.

\section{Results and discussion}

The obtained results are presented in two subtitles to discuss heat transfer through the cavity and walls.

\subsection{Heat transfer through the cavity}

The isotherms, streamlines and heatlines of the cavity with $R a=10^{6}$ for three different values of conductivity ratio $(K=0,1$ and 50$)$ are shown in Fig. 2. For $K=0$, no isotherm and heatline were drawn in the horizontal walls since no heat transfer exists in the solid. For the cavity with $K=1$, the temperature distribution in the horizontal walls is two dimensional. However, for cavity with $K=50$ temperature distribution in the horizontal walls is almost linear and heat transfer seems one dimensional. The comparison of heatlines of cavities with different $K$ shows that the increase of thermal conductivity ratio enhances heat flux between the solid and fluid. It also increases heat transfer through the solid walls. Temperature variation along the upper solid-fluid interface of the same cavity is illustrated in Fig. 3 for

a)

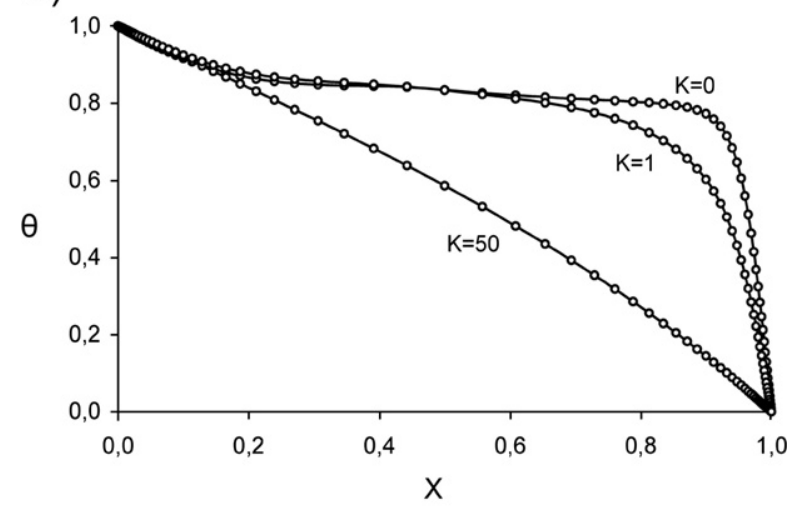

b)



Fig. 3. Temperature distribution along the upper solid-fluid interface a) $R a=10^{6}$, b) $R a=10^{3}$. 

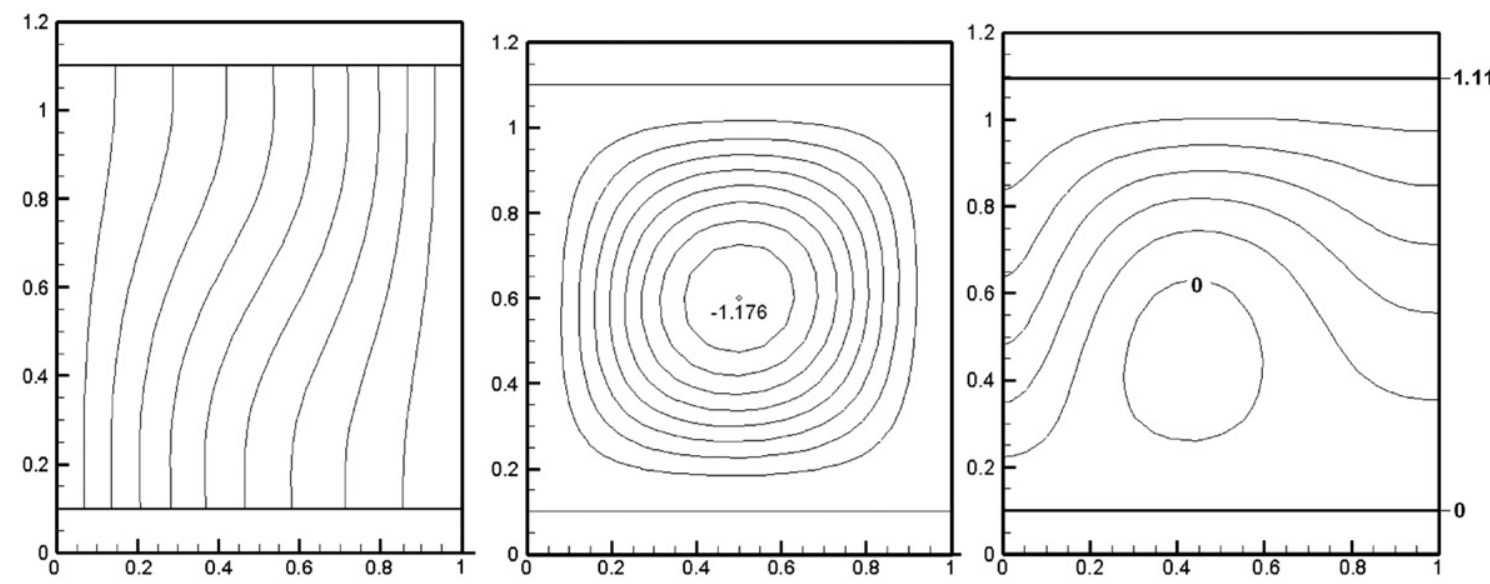

a)
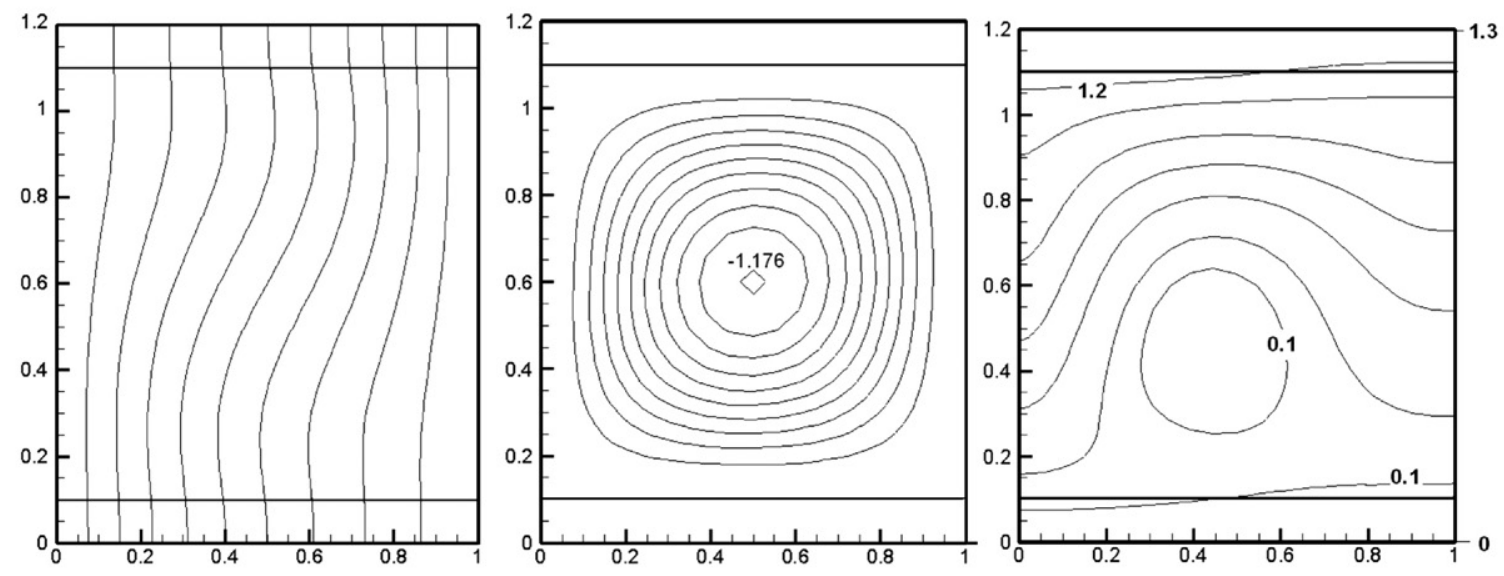

b)
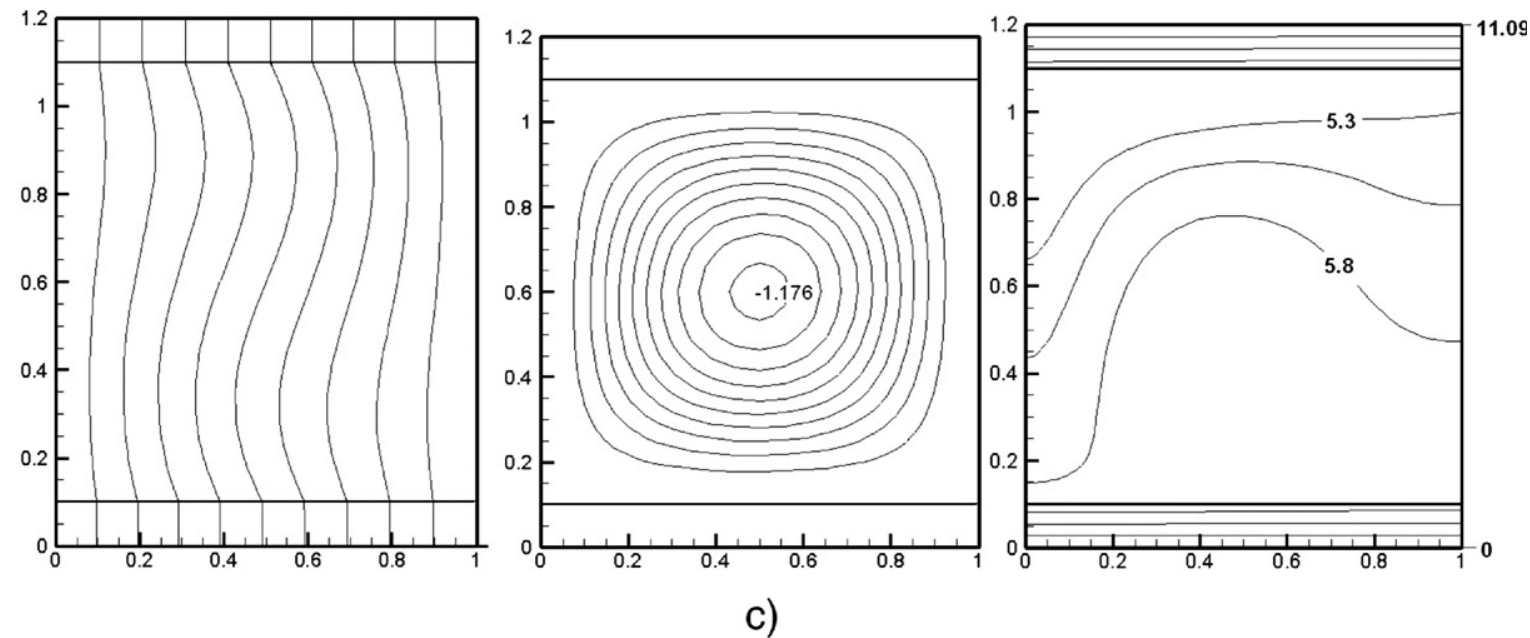

c)

Fig. 4. Isotherms, streamlines and heatlines in the cavity with $R a=10^{3}$ a) $K=0, \delta \theta=0.1, \delta \psi=0.130, \delta H=0.185$ b) $K=1, \delta \theta=0.1, \delta \psi=0.118$, $\delta H=0.22(H=1.2$ was added $)$ c) $K=50, \delta \theta=0.1, \delta \psi=0.118, \delta H=1.39(H=5.3$ and 5.8 were added $)$. 




Fig. 5. Variation of $\bar{N} u_{\mathrm{f}}$ at $X=0$ vertical wall with thermal conductivity ratio.

$R a=10^{6}$ and $R a=10^{3}$. As is seen from Fig. 3(a), for low conductivity ratio, $K=1$, the interface temperature behaves isothermally in most of the region and it is close to the interface temperature of cavity with $K=0$, however, it becomes nearly linear for $K=50$.

The isotherms, streamlines and heatlines for the cavity with $R a=10^{3}$ and for $K=0,1$ and 50 are shown in Fig. 4. Temperature distributions in the cavity and horizontal walls are considerably different than the case of $R a=10^{6}$. The isotherms in the fluid region as well as in the solid walls are nearly parallel to each other since convection heat transfer is poor in the entire fluid domain. Temperature distribution in the horizontal walls is almost one dimensional for both $K=1$ and $K=50$. One dimensional heat transfer throughout the solid walls can be observed from heatlines. Fig. 3(b) shows temperature distribution of the upper solid-fluid interface of cavity with $R a=10^{3}$. For three different values of conductivity ratio, the interface temperatures are close to each other. For the cavity with $K=50$, a linear temperature distribution on the interface exists.

The variation of average $N u$ number of vertical isothermal wall at $X=0$ with thermal conductivity ratio is shown in Fig. 5 . The average $N u$ number decreases with the increase of thermal conductivity ratio; however the rate of decrease depends on $R a$. For $R a=10^{6}$, it declines and then it almost remains constant for high values of $K$. The increase of $K$ enhances heat transfer between the horizontal walls and adjacent fluid which horizontally flows. In the upper region of cavity, a portion of heat of fluid which horizontally moves is transferred to the horizontal wall before reaching to the cold vertical wall. In the lower region of cavity, fluid which travels horizontally towards the hot vertical wall is heated by the lower horizontal wall. Thus, $\bar{N} u_{\mathrm{f}}$ decreases with increase of $K$. For further increase of thermal conductivity ratio, the rate of heat transfer between the horizontal walls and fluid is not considerably changed and as a result, $\bar{N} u_{\mathrm{f}}$ at the hot vertical wall does not vary with thermal conductivity ratio.

For the cavity with $R a=10^{3}$, the value of average $N u$ number through the cavity is not considerably affected by thermal conductivity ratio since convection heat transfer in the cavity is poor.

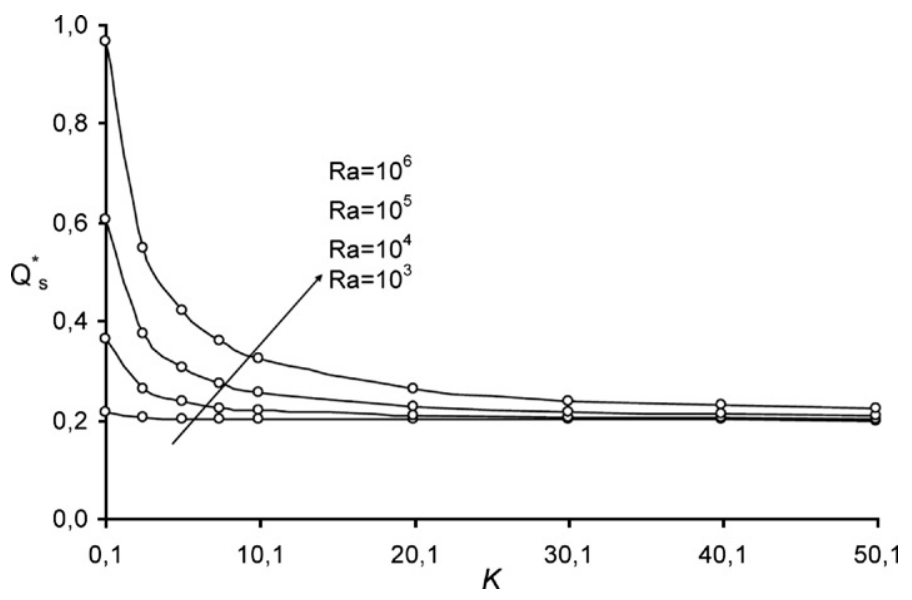

Fig. 6. The influence of thermal conductivity ratio on dimensionless heat transfer rate from lower and upper horizontal walls at $X=0$. 


\subsection{Heat transfer through the horizontal walls}

The effect of thermal conductivity on dimensionless heat transfer rate from upper and lower horizontal walls at $X=0$ surface is illustrated in Fig. 6. The value of $K$ is started by 0.1 in Fig. 6, since there is no heat transfer in the horizontal walls for the case of $K=0$. For $R a=10^{3}$, the dimensionless heat transfer rate from the horizontal walls at $X=0$ is almost constant because the convection heat transfer in the cavity is poor and heat transfer in the walls is one dimensional for different values of $K$. It approaches to 0.2 which is the dimensionless heat transfer rate from the walls with completely one dimensional conduction heat transfer. For the cavity with $R a=10^{6}$ and for small values of conductivity ratio (e.g. $K=1$ ), the dimensionless heat transfer rate decreases with increase of $K$ and it also approaches to the same value of one dimensional heat conduction $\left(Q_{\mathrm{s}}^{*}=0.2\right)$.

\section{Conclusion}

For a square cavity with finite thickness horizontal walls, the influence of $R a$ number and thermal conductivity ratio on heat transfer rate across the cavity and horizontal walls are numerically investigated. Based on the findings in this study, following results are summarized:

1) Although the horizontal wall does not directly reduce temperature gradient across the cavity, it can affect heat transfer rate through the cavity particularly in cavities with high values of $R a$ number and thermal conductivity ratio.

2) The increase of $R a$ number and conductivity ratio increase heat transfer between the wall and fluid on the solidfluid interface.

3) For the cavity with $R a=10^{6}$ and low values of conductivity ratio, natural convection heat transfer from the cavity is reduced with increase of conductivity ratio, however, heat transfer rate is almost constant for high values of thermal conductivity ratio. For $R a=10^{3}$, natural convection heat transfer across the cavity is not considerably influenced by $K$.

4) For small values of $R a$ number $\left(R a=10^{3}\right)$ or large values of conductivity ratio $(K=50)$, conduction heat transfer in the horizontal walls is almost one dimensional. For $R a=10^{6}$ and small values of conductivity ratio, the dimensionless heat transfer rate from the end of lower and upper walls decreases with the increase of thermal conductivity ratio. It approaches to a constant 0.2 value for high values of conductivity ratio. For $R a=103$, the dimensionless heat transfer rate from the end of lower and upper horizontal walls is not influenced by conductivity ratio and it is almost constant as 0.2 .

\section{References}

[1] E.V. Kalabin, M.V. Kanashina, P.T. Zubkov, Natural convection in a square cavity with time-varying side-wall temperature, Numer. Heat Transf., A Appl. 47 (6) (2005) 621-631.

[2] E.V. Kalabin, M.V. Kanashina, P.T. Zubkov, Heat transfer from the cold wall of a square cavity to the hot one by oscillatory natural convection, Numer. Heat Transf., A Appl. 47 (6) (2005) 609-619.

[3] N.H. Saeid, Y. Yaacob, Natural convection in a square cavity with spatial side-wall temperature variation, Numer. Heat Transf., A Appl. 49 (7) (2006) 683-697.

[4] A. Ben-Nakhi, A.J. Chamkha, The effect of length and inclination of a thin fin on natural convection in a square cavity, Numer. Heat Transf., A Appl. 50 (4) (2006) 389-407.

[5] Y. Varol, A. Koca, H.F. Oztop, Application of central difference scheme to the solution of natural convection equations for irregular shaped cavity enclosures, J. Appl. Sci. 7 (2007) 553-558.

[6] D.C. Lo, D.L. Young, K. Murugesan, GDQ method for natural convection in a cubic cavity using velocity-vorticity formulation, Numer. Heat Transf., B Fundam. 48 (4) (2005) 363-386.

[7] D.M. Kim, R. Viskanta, Effect of wall heat conduction on natural convection heat transfer in a square enclosure, J. Heat Transfer 107 (1985) $139-146$.

[8] D.M. Kim, R. Viskanta, Study of effects of wall conductance on natural convection in differently oriented square cavities, J. Fluid Mech. 144 (1984) 153-176.

[9] D.M. Kim, R. Viskanta, Heat transfer by conduction, natural convection and radiation across rectangular cellular structure, Int. J. Heat Fluid Flow 5 (1984) 205-212.

[10] W. Wu, D. Ewing, C.Y. Ching, The effect of the top and bottom wall temperatures on the laminar natural convection in an air-filled square cavity, Int. J. Heat Mass Transfer 49 (2006) 1999-2008.

[11] D.A. Kaminski, C. Prakash, Conjugate natural convection in a square enclosure: effect of conduction in one of the vertical walls, Int. J. Heat and Mass Transfer 12 (1986) 1979-1988.

[12] D. Misra, D.A. Sarkar, Finite element analysis of conjugate natural convection in a square enclosure with a conducting vertical wall, Comput. Methods Appl. Mech. Eng. 141 (1997) 205-219. 
[13] A. Liaqat, A.C. Baytas, Conjugate natural convection in a square cavity enclosure containing volumetric sources, Int. J. Heat and Mass Transfer 44 (2001) 3273-3280

[14] S. Acharya, C.H. Tsang, Influence of wall conduction on natural convection in an inclined square enclosure, Warme und Stoffübertragunug 21 (1987) 19-30.

[15] Z.G. Du, E. Bilgen, Coupling of wall conduction with natural convection in a rectangular enclosure, Int. J. Heat Mass Transfer 35 (1992) $1962-1975$.

[16] R.B. Yedder, E. Bilgen, Laminar natural convection in inclined enclosures bounded by a solid wall, Heat Mass transfer 32 (1997) $455-462$.

[17] N.H. Saeid, Conjugate natural convection in a porous enclosure: effect of conduction in one of the vertical walls, Int J Therm. Sciences 46 (2007) 531-539.

[18] A.C. Baytas, A. Liaqat, T. Grosan, V. Pop, Conjugate natural convection in a square cavity, Heat Mass Transfer 37 (2001) $467-473$.

[19] W.J. Chang, H.C. Lin, Wall heat conduction effect on natural convection in an enclosure filled with a non-Darcian porous medium, Numer. Heat Transf., A Appl. 26 (1994) 671-684.

[20] V.A.F Costa, Bejan's heatlines and masslines for convection visualization and analysis, Appl. Math. Rev. 59 (2006) 126-145.

[21] Y. Jaluria, K. Torrence, Computational Heat Transfer, 2nd edn. Taylor and Francis, 2003.

[22] M. Mobedi, Y. Yuncu, A three dimensional numerical study on natural convection heat transfer from short horizontal rectangular fin array, Heat Mass Transfer 39 (2003) 267-275.

[23] K.L. Wong, A.J. Baker, A 3D incompressible Navier-Stokes velocity vorticity weak form of finite element algorithm, Int. J. Num. Meth. Fluids 38 (2002) 99-123.

[24] G.D. Vahl Davis, Natural convection of air in a square cavity; a bench mark numerical solution, Int.J. Num. Meth. Fluids 3 (1983) $249-264$. 